\title{
Unfolding of $\alpha$-helical 20-residue poly-glutamic acid analyzed by multiple runs of canonical molecular dynamics simulations
}

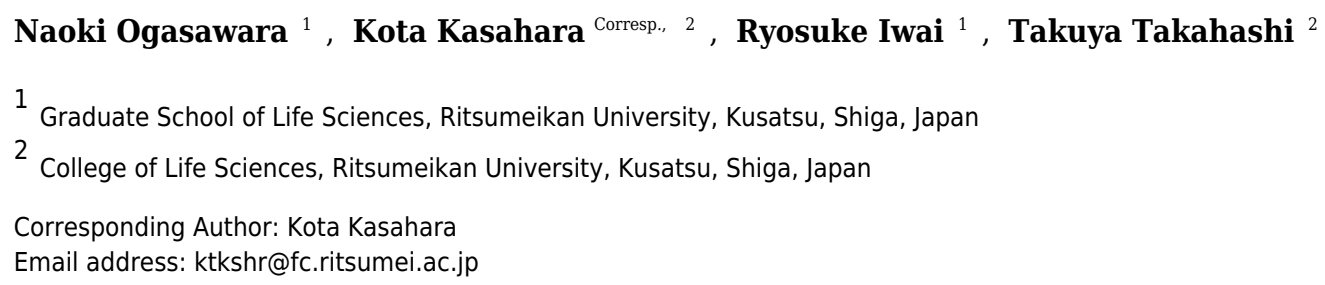

Elucidating the molecular mechanism of helix-coil transitions of short peptides is a longstanding conundrum in physical chemistry. Although the helix-coil transitions of polyglutamic acid (PGA) have been extensively studied, the molecular details of its unfolding process still remain unclear. We performed all-atom canonical molecular dynamics (MD) simulations for a 20-residue PGA, over a total of $19 \mu \mathrm{s}$, in order to investigate its helixunfolding processes in atomic resolution. Among the 28 simulations, starting with the $\alpha$ helical conformation, all showed an unfolding process triggered by the unwinding of terminal residues, rather than by kinking and unwinding of the middle region of the chain. The helix-coil-helix conformation which is speculated by the previous experiments was not observed. Upon comparison between the $\mathrm{N}$ - and C-termini, the latter tended to be unstable and easily unfolded. While the probabilities of helix elongation were almost the same among the N-terminal, middle, and C-terminal regions of the chain, unwinding of the helix was enriched at the $\mathrm{C}$-terminal region. The turn and $3_{10}$-helix conformations were kinetic intermediates in the formation and deformation of $\alpha$-helix, consistent with the previous computational studies for Ala-based peptides. 


\section{Unfolding of $\alpha$-helical 20-residue poly-glutamic acid}

\section{2 analyzed by multiple runs of canonical molecular}

\section{3 dynamics simulations}

4 Naoki Ogasawara ${ }^{1,+}$, Kota Kasahara ${ }^{2,+, *}$, Ryosuke Iwai ${ }^{1}$, Takuya Takahashi ${ }^{2}$

$5{ }^{1}$ Graduate School of Life Sciences, Ritsumeikan University, Noji-Higashi 1-1-1, Kusatsu, Shiga,

$6 \quad 525-8577$, Japan

$7 \quad{ }^{2}$ College of Life Sciences, Ritsumeikan University, Noji-Higashi 1-1-1, Kusatsu, Shiga, 525-

8 8577, Japan

$9 *$ To whom correspondence should be addressed:

10 Kota Kasahara, Tel: +81-77-566-1111; Fax: +81-77-561-3729; Email: ktkshr@fc.ritsumei.ac.jp.

$11 \uparrow$ The first two authors are considered to be joint first authors. 


\section{Abstract}

14 Elucidating the molecular mechanism of helix-coil transitions of short peptides is a long-

15 standing conundrum in physical chemistry. Although the helix-coil transitions of poly-glutamic

16 acid (PGA) have been extensively studied, the molecular details of its unfolding process still

17 remain unclear. We performed all-atom canonical molecular dynamics (MD) simulations for a

18 20-residue PGA, over a total of $19 \mu$ s, in order to investigate its helix-unfolding processes in

19 atomic resolution. Among the 28 simulations, starting with the $\alpha$-helical conformation, all

20 showed an unfolding process triggered by the unwinding of terminal residues, rather than by

21 kinking and unwinding of the middle region of the chain. The helix-coil-helix conformation

22 which is speculated by the previous experiments was not observed. Upon comparison between

23 the $\mathrm{N}$ - and $\mathrm{C}$-termini, the latter tended to be unstable and easily unfolded. While the probabilities

24 of helix elongation were almost the same among the N-terminal, middle, and C-terminal regions

25 of the chain, unwinding of the helix was enriched at the C-terminal region. The turn and $3_{10}$-helix

26 conformations were kinetic intermediates in the formation and deformation of $\alpha$-helix, consistent

27 with the previous computational studies for Ala-based peptides.

\section{Introduction}

31 Elucidation of the molecular mechanisms of protein folding is a central issue in physical

32 chemistry. Since protein folding involves formation of secondary structural elements as building

33 blocks of the tertiary structure (Richardson, 1981), understanding the dynamics of $\alpha$-helical

34 folding and unfolding, or helix-coil transition, is essential. The helix-coil transition has been 
35 extensively studied in both experimental and theoretical methods using mainly Ala-based 36 polypeptides (Baldwin, 1995; Chen, Zhou \& Ding, 2007; Neumaier et al., 2013) due to the high

37 helix propensity of Ala residues (Spek et al., 1999). Another representative model peptide is 38 poly-glutamic acid (PGA). Since the side-chain of Glu has a titratable group, the chemical nature 39 of PGA can be modulated by the solution $\mathrm{pH}$, and its helix-coil equilibrium can be controlled by 40 pH adjustments (Nakamura \& Wada, 1981; Clarke et al., 1999; Kimura et al., 2002; Inoue, 41 Baden \& Terazima, 2005; Causgrove \& Dyer, 2006; Finke et al., 2007; Stanley \& Strey, 2008; 42 Donten \& Hamm, 2013; Gooding et al., 2013). Previous experiments on the helix-coil 43 transitions of PGA reported that compared to neutral environments, acidic environments enhance 44 helix formation. The reported helix content of short PGAs in acidic environments varied from 450.3 to 0.6 , whereas it is below detectable limit in neutral pH (Clarke et al., 1999; Kimura et al., 46 2002; Finke et al., 2007). Detailed scenario of the dynamics of helix-coil transitions is still 47 controversial. The previous reports have presented two different types of PGA conformations in acidic environments: (i) a single $\alpha$-helix with denatured termini and (ii) multiple short $\alpha$-helices connected by coil regions. Kimura et al. proposed that the single $\alpha$-helical conformation arises 50 via intermediate states with several short helices, based on Fourier-transform infra-red (FTIR) spectroscopy and circular dichroism (CD) experiments (Kimura et al., 2002). Clarke et al. 52 implied, based on stopped-flow CD measurements, that the single long $\alpha$-helical conformation 53 successively decomposes into multi-helical conformations (Clarke et al., 1999). Finke et al. 54 supported this scenario based on fluorescence resonance energy transfer (FRET) measurements 55 (Finke et al., 2007). In order to shed light on peptide conformational transitions at the atomic level, molecular dynamics (MD) simulation is a promising approach. This method has been applied to investigate 
58 the helix-coil transitions of Ala-based peptides, and the C-terminus has been reported to have a

59 higher denaturing tendency compared to the N-terminus (Young \& Brooks, 1996; Takano et al., 60 1999; Wu \& Wang, 2001). In addition, the $3_{10}$-helix and turn conformations were found to be

61 kinetic intermediates for the helix-coil transitions (Young \& Brooks, 1996; Takano et al., 1999).

62 However, unlike that of the Ala-based peptides, helix-coil transitions of PGA peptides have not

63 been studied using the all-atom MD method.

Here, we utilized the all-atom canonical MD method to simulate unfolding dynamics of a 20-residue PGA with fully protonated side chains, mimicking an acidic environment. Using the molecular model of a PGA with $\alpha$-helical conformation as the initial structure, we repeated MD

67 simulations for unfolding processes with different initial conditions. In total, 19- $\mu$ s dynamics, consisting of 3 runs with $3.0 \mu$ s and 25 runs with $0.4 \mu \mathrm{s}$, were simulated. While various pathways

69 of unfolding were observed in these 28 time courses, PGA unfolding was mainly seen to be triggered by denaturation of the termini, followed by propagation of the coil conformation

71 toward the opposite side. Multiple-helix conformations implied by the previous experiments did 72 not appear in the MD simulations.

\section{Methods}

\section{Canonical MD Simulations}

76 Dynamics of a 20-residue PGA, in an explicitly solvated periodic boundary cell, was

77 investigated by the canonical MD method. We prepared two $\alpha$-helical PGA structures as the 78 initial structures for simulation. The first was an $\alpha$-helical structure, sampled from an ensemble, 
79 obtained by our replica-exchange MD (REMD) simulation, with an implicit solvent model. The

80 details of the REMD simulation will be described elsewhere (unpublished data by Iwai R et al.).

81 The second was an ideal $\alpha$-helix, all the residues of which took the backbone dihedral angles $\varphi=$ $82-60^{\circ}$ and $\psi=-45^{\circ}$, built using $t L E a P$ software attached to AMBER package. The N- and C83 termini of the PGA were capped with acetyl (Ace) and N-methyl (Nme) groups, respectively. All 84 the carboxyl groups of the side-chains were protonated and net charge of the PGA was zero. 85 Each molecular model of the PGA was placed in the truncated octahedral cell and solvated by 86 filling with TIP3P water molecules (Jorgensen et al., 1983). The number of atoms composing the 87 molecular system with the simulated structure of PGA was 10,592 , and that with the ideal $\alpha$ 88 helix was 11,081. After that, the energy minimizations were successively performed with the 89 steepest descent and conjugate gradient methods; the number of steps was 250 for each. The 90 systems were relaxed via a 200-ps NPT simulation using Berendsen barostat. For the system with 91 the ideal helix, the heavy atoms in the PGA were constrained during the relaxation run. The final 92 snapshots of these two systems, referred to as Sim and Ide, were used as the initial structures of 93 the production runs (Figure 1). Through the NPT relaxations, the cell dimensions shrank from $9454.32 \AA$ to $51.85 \AA$ and from $55.10 \AA$ to $52.66 \AA$ for $\operatorname{Sim}$ and Ide, respectively. The convergence 95 of cell volumes was conformed in terms of the relative standard deviations in the last 100 ps of 96 the NPT simulations ( $c a .0 .23 \%$ ). As production runs, 8 and 20 runs of simulations were 97 performed with Sim and Ide systems, respectively. Accordingly, we termed these simulations as $98 \operatorname{Sim} 1, \operatorname{Sim} 2, \ldots, \operatorname{Sim} 8$, and $I d e 1, I d e 2, \ldots, I d e 20$. The initial atomic velocities were randomly 99 generated with different random seeds for each run. The simulation time of each run was $0.4 \mu \mathrm{s}$ 100 except for $\operatorname{Sim} 1, \operatorname{Sim} 2$, and $\operatorname{Sim} 3$ that lasted over $3.0 \mu$ s. These production runs were performed 101 with the $N V T$ ensemble at $300 \mathrm{~K}$ using the Langevin thermostat. The integration time step was 
$1022.0 \mathrm{fs}$; the covalent-bond lengths with hydrogen atoms were constrained with the SHAKE

103 algorithm (Ryckaert, Ciccotti \& Berendsen, 1977). The non-bonded pairwise potentials were

104 truncated at $10 \AA$ of the interatomic distance. For the potential energy calculations, AMBER

105 ff99SB force field (Hornak et al., 2006) was applied. All the simulations were carried out using

106 AMBER software.

107

108 Analyses

109 On the basis of the trajectories of the atomic coordinates, recorded every 20 ps in the

110 simulations, the helix-coil transitions of a PGA were analyzed using DSSP software (Kabsch \&

111 Sander, 1983). DSSP recognizes the secondary structural elements in terms of hydrogen bonding

112 patterns of the main-chains and categorizes them into the following eight classes: $\alpha$-helix, $3_{10^{-}}$

113 helix, $\pi$-helix, extended $\beta$-strand, isolated $\beta$-bridge, turn, bend, and others. Each class is

114 represented by an alphabetical symbol; $H, G, I, E, B, T, S$, and $O$, respectively. Note that the

115 symbol ' $O$ ' is introduced in this paper for convenience, and it is denoted as ', (white or blank

116 space) in the output of the DSSP software. The secondary structure content in the Ide trajectories

117 was referred to as $P^{I d e}(x ; i)$ for the contents of the secondary structure $x$ (any of the eight classes)

118 at the $i$-th residue. The superscript "Ide" indicates that the ensemble was obtained from the 20

119 Ide runs with $0.4 \mu$ s each. The ensemble consisting of trajectories of 8 Sim runs with $0.4 \mu$ s each

120 is indicated as the superscript "Sim", and that of $\operatorname{Sim} 1-\operatorname{Sim} 3$ with $3.0 \mu$ s each is indicated as the

121 superscript "Sim1-3". The secondary structure content for the entire chain is presented as $P^{\text {Ide }}(x)$.

122 The transition probabilities of $i$-th residue, from the secondary structure $x$ to $y$ between the

123 successive snapshots (20 ps of the time interval), $P^{I d e}(y, x ; i)$, were also evaluated. To measure the 
124 time required for the complete unfolding of an $\alpha$-helix, we defined the unfolding time, $t_{\mathrm{u}}$, as the

125 time corresponding to the first snapshot without $\alpha$-helical residues in a trajectory.

126

127 Results

128 Micro-second Dynamics of a PGA

129 In order to investigate long-term behavior of a PGA, we performed three runs of 3.0- $\mu$ s MD

130 simulations ( $\operatorname{Sim} 1, \operatorname{Sim} 2$, and $\operatorname{Sim} 3)$ with the same initial atomic coordinates but different atomic

131 velocities (Figure 1). The initially formed $\alpha$-helix was deformed immediately after beginning the

132 simulations in all the three runs (Figure 2). The unfolding times, $t_{\mathrm{u}}$, defined as the time of the

133 first snapshot without an $\alpha$-helical residue in PGA for each trajectory, were $31.06 \mathrm{~ns}, 100.52 \mathrm{~ns}$,

134 and $7.38 \mathrm{~ns}$ in $\operatorname{Sim} 1, \operatorname{Sim} 2$, and $\operatorname{Sim} 3$ simulations, respectively. In the simulation with the longest

135 unfolding time (Sim2), after unfolding of the initial $\alpha$-helix, the helical conformation was

136 temporarily reformed at the $\mathrm{N}$-terminal half of the chain at around $0.2 \mu$ s (Figure $2 \mathrm{G}$ ). However,

137 the reformed helix was unfolded at $0.34 \mu \mathrm{s}$, and a helix longer than 13 residues was not formed

138 till the end. In the Sim 1 simulation, although the initial helix was immediately unfolded, a long

139 helix consisting of 17 residues was refolded and retained over sub-micro second time scale

140 (Figure 2E). This helix was nucleated between 12th to 16 th residues at $0.62 \mu$ s (Figure 2D) and

141 propagated over the range from 2 nd to 18 th residues. While the N-terminal half of the helix was

142 deformed at $0.84 \mu$ s (Figure $2 \mathrm{~F}$ ), the latter half remained intact till $0.95 \mu \mathrm{s}$. On the other hand, re-

143 formation of stable helix did not occur in Sim3, although several helix-nucleation events were

144 observed. Overall, helix formation was a relatively rare event in this time scale. In addition, 145 while several helix-nucleation events were observed, the nucleated helices disappeared 
146 immediately in most cases. Helix nucleation seemed to be coupled with the turn conformation

147 (Figures 2A, B, and C), discussion on which will be taken up later. Formation of a $\beta$-sheet was

148 also observed as a rare event. $\beta$-sheet formation in Sim2 was exceptionally stable and was

149 retained during $0.63 \mu$ s (Figure $2 \mathrm{H})$.

150 In the time course of the secondary structural elements at each residue (Figure $2 \mathrm{~A}, \mathrm{~B}$, and

151 C), some "bands" could be observed; for example, the turn conformation was almost always

152 formed at the 9th and 10th residues in Sim2. Since the tendency to form a turn at the 9th and 10th

153 residues was not observed in the other runs, it is considered to be due to an initial condition,

154 rather than an intrinsic propensity of the 9th and 10th residues. This indicates that there was the

155 strong time-correlation of secondary structure formation, and $3.0 \mu$ s was not enough to reach an

156 equilibrium state. The time course of the ensemble average of the helix content (summation over

157 the $\alpha$ - and $3{ }_{10}$-helix conformations; $P(H)+P(G)$ ), for Sim1, Sim2, and Sim3 implies that the

158 trajectories were not well-converged (Figure 3). The gain of helix content in $0.5-1.0 \mu$ s of Sim 1

159 corresponds to the refolding of the $\alpha$-helix mentioned in the previous paragraph (Figure 2A, D, E,

$160 \mathrm{~F})$. While the helix content of the three trajectories became converged to similar values with the

161 evolution of time, they still acquired different values at the end of the simulations. The helix

162 content in the full-length trajectories of $\operatorname{Sim} 1, \operatorname{Sim} 2$, and $\operatorname{Sim} 3$ were 0.14, 0.12, and 0.078,

163 respectively. In addition, the time courses of the end-to-end distance and radius of gyration also

164 showed slow equilibrations of the conformations (Figure S1). These results imply that 165 equilibration of the system requires longer time scales.

166

167 Unfolding Dynamics 
168 Non-equilibrium processes involved in the transformation of an $\alpha$-helix into denatured structures

169 were analyzed by scrutinizing the first part of each trajectory. We additionally performed 25

170 short (400 ns for each) simulations and analyzed the unfolding processes of the 28 simulations in

171 total. Note that 8 of them started from an $\alpha$-helical conformation obtained from a simulation

172 (Sim1-Sim8; Figure 1), and the remaining 20 started from an artificially built ideal $\alpha$-helix (Ide 1-

173 Ide20; Figure 1). As a result, all the 28 runs showed corruption of the $\alpha$-helical conformation

174 within $400 \mathrm{~ns}$ (Figures 4 and 5). The unfolding times $\left(t_{\mathrm{u}}\right)$ varied from $7.38 \mathrm{~ns}(\operatorname{Sim} 3)$ to $380.70 \mathrm{~ns}$

175 (Sim6), and the average, median, and the standard deviation (SD) were $75.63 \mathrm{~ns}, 36.02 \mathrm{~ns}$, and

17692.18 ns, respectively (Table 1). There was no statistically significant difference between Sim

177 and Ide simulation results; the average (median; SD) of $t_{\mathrm{u}}$ were $72.65 \mathrm{~ns}(36.02 \mathrm{~ns} ; 79.88 \mathrm{~ns})$ and

$17883.09 \mathrm{~ns}(37.32 \mathrm{~ns} ; 123.97 \mathrm{~ns})$, for Sim and Ide, respectively.

179 The unfolding trajectories varied among the 28 trajectories. The fastest unfolding was

180 observed in Sim3. The helix deformed from both the N- and C-termini immediately after the

181 simulation began (Figure 5C). As described above, while a single-turn helix sometimes formed

182 at the $\mathrm{N}$ - and C-termini after unfolding, they did not grow into a longer helix. The bend

183 conformations were stably formed at the 5th, 6th, 7th, 10th, and 11 th residues during $400 \mathrm{~ns}$. On

184 the contrary, Sim6 showed the slowest dynamics of unfolding. While three or four residues from

185 the N-terminus were immediately deformed, the remaining part of the helix was retained for a

186 long time (Figure 5F). As described above, strong time correlations were observed in all the

187 trajectories (Figures 4 and 5). After immediate unfolding of the $\alpha$-helix, a denatured

188 conformation of the peptide was not randomized in this time scale.

For all the 28 trajectories, unfolding mechanisms were analyzed in terms of the order of 
191 regions; i.e., the $\mathrm{N}$-terminal region (2nd-7th residues), the middle region (8th-13th residues),

192 and the C-terminal region (14th-19th residues). The first and 20th residues were discarded

193 because of the following reasons: they would be highly influenced by the truncation of the chain;

194 the main-chain hydrogen bonding pattern of the first residue cannot be defined due to lack of the

195 N-terminal neighbor; all the regions should have the same number of residues. Next, the order of

196 unfolding, for these regions, was assessed based on the helix content of each region in the time

197 period ranging from the beginning of simulation to the unfolding time, $t_{\mathrm{u}}$. As a result, the

198 unfolding process beginning with the deformation of the middle region was not observed, and all

199 the unfolding processes began with unwinding of one of the terminal regions (Table 1). In

200 addition, coil regions propagated toward both the directions in many cases. There are two

201 possible scenarios for completion of unfolding from any terminus: (i) the coil region appears in a

202 terminus and elongates toward the opposite terminus ("N, M, C" and "C, M, N" in Table 1), and

203 (ii) the opposite terminus is successively unfolded followed by elongation of coil regions from

204 both the termini to the middle ("N, C, M" and "C, N, M" in Table 1). The fact that the former

205 scenario was observed in only 3 and 2 runs among 20 Ide and 8 Sim runs, respectively, suggests

206 the latter being the major way of $\alpha$-helix unfolding in this system.

When comparing the $\mathrm{N}$ - and $\mathrm{C}$-termini of the peptide chain, unfolding from the $\mathrm{C}$ terminus was preferred over that from the N-terminus; 13 out of the 20 Ide runs and 7 out of the $8 \mathrm{Sim}$ runs showed unfolding from the C-terminus. Difference between the two termini was clearer in Sim runs than in Ide runs, probably because of the slightly distorted initial structure of

$211 \operatorname{Sim}$ (Figure 1). The ensemble averages of residue-wise $\alpha$-helix contents in Ide $1-20$ with $0.4 \mu \mathrm{s}$

212 each $\left(P^{I d e}(H ; i)\right)$, Sim 1-8 with $0.4 \mu$ s each $\left(P^{\operatorname{Sim}}(H ; i)\right)$, and $\operatorname{Sim} 1-3$ with $3.0 \mu$ s each $\left(P^{\operatorname{Sim} 1-3}(H ; i)\right)$

213 also showed a lower helical tendency at the C-terminus than at the N-terminus (Figure 6). The 
214 previous simulation studies (Young \& Brooks, 1996; Wu \& Wang, 2001; Finke et al., 2007) had

215 also reported that helix formation of the C-terminal residues was unstable compared to that of the

216 N-terminal ones.

217

218 Secondary Structural Transitions

219 To analyze the detailed mechanisms of conformational transitions in shorter time scales, we

220 assessed the probability of the event that the $i$-th residue in the secondary structure $A$ at time $t$ is

221 transformed into $B$ at time $t+20 \mathrm{ps}$; the averaged probability over the 20 Ide runs is referred to

222 as $P^{I d e}(B, A ; i)$. For simplicity, we focused on the four classes of secondary structure elements; $H$,

$223 G, T$, and $\overline{H G T}$, which means any of the other five structural elements $(I, E, S, B$, and $O)$. The

224 cases $i=2,11$, and 19 were analyzed as representatives of the $\mathrm{N}$-terminal, middle, and C-

225 terminal residues, respectively (Table 2). The C-terminal residues showed a weaker tendency to

226 retain the $\alpha$-helical conformation compared to the other residues $\left(P^{I d e}(H, H ; 2)=0.94, P^{I d e}(H, H ; 11)\right.$

$227=0.96$, and $\left.P^{I d e}(H, H ; 19)=0.58\right)$. The weaker tendency to retain the same conformation in the C-

228 terminal region was also observed in the other secondary structures. The results of Sim runs were

229 qualitatively consistent with that of Ide runs (Table S1).

230

The helix-coil transitions mainly occurred via the turn conformation. More than half of

231 the conformational transitions from the $\alpha$-helix directed to the turn conformation; $P^{I d e}(T, H ; i) / P^{I d e}($

$232 \bar{H}, H ; i)$ for $i=2,11$, and 19 were $0.52,0.73$, and 0.80 , respectively, where $\bar{H}$ denotes the

233 secondary structure other than $H$. In addition, formation of the $\alpha$-helix via turn was enriched in

234 the C-terminal residue; $P^{I d e}(H, T ; i) / P^{I d e}(\bar{T}, T ; i)$ for $i=2,11$, and 19 were $0.19,0.62$, and 0.42 ,

235 respectively. Thus, the turn conformation can be considered as an intermediate state in the helix- 
236 coil transition, especially at the C-terminus. Another intermediate in the $\alpha$-helix formation is the

2373 -helix. While a major destination state of a $3_{10}$-helix was the turn $\left(P^{I d e}(T, G ; i) / P^{I d e}(\bar{G}, G ; i)\right.$ for $i=$

2382,11 , and 19 were $0.55,0.62$, and 0.64 , respectively), it also transformed into an $\alpha$-helix,

239 especially at the middle position; $P^{I d e}(H, G ; i) / P^{I d e}(\bar{G}, G ; i)$ for $i=2,11$, and 19 were $0.20,0.34$, and

240 0.076, respectively (Supplementary Figure S2). This result agreed with the previous theoretical

241 studies, which reported that the $3_{10}$-helix is not a thermodynamic intermediate but could be a

242 kinetic intermediate (Young \& Brooks, 1996; Wu \& Wang, 2001).

ends was analyzed. We focused on segments consisting of three consecutive residues in the chain,

and the state of the segment was defined as the combination of secondary structures of the three residues, grouped into the two classes, i.e., $\alpha$-helix (" $H$ ") and others ("-"; it has the same meaning as " $\bar{H}$ "). The state of a segment was divided into the following eight classes: " $H H H$ ", " $H H-"$, “-HH”, " $H-H ”$, " $H--$ ", “--H”, and "---". The state " $-H-"$ is impossible, because $\alpha$-helical conformation coincides with at least four consecutive residues. The probability of the event that the central residue of a segment forms an $\alpha$-helix at the next snapshot (20 ps later) was analyzed for each class. For instance, probability for the class " $H H_{-}$", denoted as $P^{I d e}(H, H H-)$, means the probability to retain $\alpha$-helical conformation for the residue at the C-terminal end of an $\alpha$-helix, regardless of the position in the chain $(i)$. The probability of deformation of the C-terminal end of an $\alpha$-helix can be shown as $P^{I d e}\left(-, H H_{-}\right)=1-P^{I d e}(H, H H-)$. The probabilities are summarized in Table 3; the case of Sim runs is shown in Table S2. We found that a residue at the interior of an $\alpha$-helix was more stable to maintain the $\alpha$-helical conformation, compared to the terminal 257 residues; $P^{I d e}(H, H H H)=0.97$. It is noteworthy that the C-terminal end of an $\alpha$-helix is more 258 frequently deformed than the N-terminal one; $P^{I d e}\left(H, H H_{-}\right)=0.74$ and $P^{I d e}(H,-H H)=0.92$. In 
259 addition, $\alpha$-helix elongation toward the C-terminus was enriched compared to that toward the 260 opposite direction; $P^{I d e}(H, H--)=0.23$ and $P^{I d e}(H,--H)=0.04$. The C-terminal end of an $\alpha$-helix

261 unstably changed its conformation while the N-terminal end tended to retain its conformation.

We also evaluated the relationship between the two definitions of position; position in an

263

264

$\alpha$-helix (the N-terminal end, internal, and the C-terminal end) and position in the polypeptide chain (the $\mathrm{N}$-terminal region $[2 \leq i \leq 7]$, middle region $[8 \leq i \leq 13]$, and C-terminal region $[14 \leq i$ $\leq 19]$ ). The probability of helix-coil transitions in the center of a three-residue segment $x$ was assessed for each of the three regions $y: P^{I d e}(-, x ; y)=0.04$, where $x$ is " $H H-$ " or " $-H H$ " for the Cand N-terminal ends of an $\alpha$-helix, respectively, and $y$ is any of " $N$ ", " $M$ ", and " $C$ ", for the Nterminal, middle, and C-terminal regions, respectively. The probabilities to unfold the $\mathrm{N}$ - and $\mathrm{C}$ terminal ends of an $\alpha$-helix varied with respect to the position of the ends in the entire chain; namely, higher probabilities were observed in the C-terminal region of the peptide chain $\left(P^{I d e}\right.$ $(-, H H-; C)>P^{I d e}(-, H H-; N)$ and $P^{I d e}(-,-H H ; C)>P^{I d e}(-,-H H ; N)$ in Table 3$)$. While residue-wise $\alpha-$ helical content (Figure 6) and $\alpha$-helix retention probability (Table 2) indicate the highest $\alpha$ helical propensity for the middle region, the lowest probabilities to unfold the ends of $\alpha$-helix were found in the $\mathrm{N}$-terminal region. In contrast, probabilities for elongation of an $\alpha$-helix were almost the same for all the three regions (see $P^{I d e}(H, H--)$ and $P^{I d e}(H,--H)$ in Table 3$)$. Therefore, an $\alpha$-helical PGA tended to unfold from the C-terminus.

On the other hand, the $\alpha$-helix nucleation was observed in low probabilities regardless of positions in the chain; $P^{I d e}(H,---)=0.02$ for all three regions.

\section{Discussion}


281 In this study, we examined the dynamics of a 20-residue PGA with 28 runs of all-atom canonical

282 MD simulations. While three of them simulated 3.0- $\mu$ s time courses, the systems were not well-

283 equilibrated (Figure 3) and complete refolding of the $\alpha$-helix was not observed (Figures 4 and 5).

284 The time scale required for $\alpha$-helix formation by PGA, still remains controversial. The suggested

285 time-scale varies from sub-micro to milliseconds (Clarke et al., 1999; Kimura et al., 2002;

286 Causgrove \& Dyer, 2006; Qin et al., 2014). Our simulation results imply that a time range of few

287 micro-seconds is too short to refold PGA in acidic environments.

We mainly focused on the non-equilibrium dynamics of unfolding processes and repeated

28 runs of simulations with the two different initial $\alpha$-helical structures. The results from these

two initial structures were qualitatively similar. Higher stability of the $\alpha$-helical conformation

was shown to be in the middle of the polypeptide chain than at the termini. All the unfolding

processes of the $\alpha$-helix began from a terminus, but a helix-coil-helix conformation was not

stably observed. In many cases, the unfolding proceeded toward both directions, rather than

starting from a terminus and ending at the opposite. In addition, unfolding from the C-terminal

side was preferred over that from the $\mathrm{N}$-terminal side (Table 1). The probability of retention of $\alpha$ helix at each residue was lower in the C-terminus than in the N-terminus (Table 2). While the probabilities of $\alpha$-helix elongation were almost the same irrespective of whether the end was unwinding of the $\alpha$-helix tended to be higher at the C-terminus of the chain (Table 3 ). The instability of $\alpha$-helix at the C-terminus was due to the enhancement of unfolding, rather than 301 reduction of folding. In the process of folding and unfolding of the $\alpha$-helices, the turn and $3_{10^{-}}$

302 helix conformations can be kinetic intermediates as consistent to the precedent studies (Young \& 303 Brooks, 1996; Wu \& Wang, 2001; Pal, Chakrabarti \& Basu, 2003). 
305 consideration. First, treatment of denatured proteins has not been fully validated in current force

306 fields. Underestimation of the radius of gyration of denatured proteins by standard force fields

307 and water models has been previously reported (Piana, Klepeis \& Shaw, 2014). While there is no

308 gold standard yet, some improved force fields and water models have been proposed to simulate

309 denatured proteins (Piana et al., 2015; Henriques \& Skepö, 2016; Huang et al., 2016). Second,

310 although the force field applied here, AMBER ff99SB, is one of the standard force fields, there

311 are some reports about its weakness; e.g., underestimation of helix stability (Sorin \& Pande,

312 2005) and discrepancy with the quantum mechanical calculations (Takano, Kusaka \& Nakamura,

313 2016). Third, finite-size effects have been reported for the helix-stability of a model polypeptide

314 (Weber, Hünenberger \& McCammon, 2000; Kastenholz \& Hünenberger, 2004; Reif et al., 2009;

315 Kasahara, Sakuraba \& Fukuda, 2018). To avoid this problem, we used the large periodic

316 boundary cells, which have at least a $10-\AA$ margin between the solute termini and the cell

317 boundaries, and the cell size was well equilibrated via the NPT simulations.

318 In fact, helix content in the simulated ensembles (Figures 3 and 6) were lower than the

319 experimentally reported values, which is in the range of 0.3 to 0.6 . The ensemble averages [and

320 SD] of end-to-end distances (19.11 [8.17], 19.57 [7.61], and 15.71 [5.85] $\AA$ for Ide, Sim, and

321 Sim 1-3, respectively) were inconsistent with the FRET measurements by Finke et al., which

322 were 23-24 $\AA$ at pH 4 (Finke et al., 2007). However, differences in the experimental method and

323 conditions may cause differences in the helix content (Kimura et al., 2002), since precise

324 measurement of the latter for short peptides is not straight forward (Kelly, Jess \& Price, 2005;

325 Greenfield, 2007). Discussion on the quantitative aspects of the results, e.g., helix contents and

326 folding kinetics, provided by both the experimental and theoretical methods in this study, should 
327 be carefully considered. From qualitative aspects, our results were consistent with the reported

328 theoretical studies, in spite of several differences in the materials and methods, e.g., peptide

329 sequence, parameters, and sampling methods. For example, the weaker helix formation

330 propensity at the C-terminus and the kinetic intermediates of helix-coil transitions were

331 consistently concluded from this study in agreement with the previous theoretical studies. They

332 are robust conclusions, regardless of adjustable settings and simulation methods. In addition to

333 that, our simulation results provide statistics of kinetic details of helix-coil transition by multiple

334 runs of canonical MD. The weaker helix formation propensity at the C-terminus is due to high

335 frequency of unwinding rather than disfavoring of folding. Helix-coil-helix conformations

336 speculated by previous experiments were not observed.

337 Note that effects of peptide length, which is one of the most important determinants of

338 the helix-coil transitions of polypeptide, is not analyzed in this study. In general, the microscopic

339 behavior of peptides depends on peptide length (Gómez-Sicilia et al., 2015). While this study

340 focused only on the behavior of 20-residue PGA by following the previous study by Finke et al

341 (Finke et al., 2007), some other previous experiments reported the effects of the length of PGA;

342 For example, Clarke et al. examined 34-, 57-, and 163-residue PGAs (Clarke et al., 1999),

343 Kimura et al. used 34- and 190-residue PGAs (Kimura et al., 2002), and Donten and Hamm used

344 20-, 50-, and 440-residue PGAs (Donten \& Hamm, 2013). They demonstrated that longer PGAs

345 tend to have slower folding kinetics and higher helix contents. For future works, simulating

346 systems with longer PGAs would be useful for understanding the molecular mechanisms of

347 effects of peptide length. 


\section{Conclusion}

350 In this study, the unfolding mechanism of $\alpha$-helix in 20-residue PGA was investigated using all-

351 atom canonical MD simulations. Our results suggested that the unfolding was triggered by

352 unwinding of a terminus, whereas the multiple short-helical conformations, implied in the

353 previous experiments (Clarke et al., 1999; Kimura et al., 2002; Finke et al., 2007), were not

354 stably observed in the simulated trajectories within the micro-second time-scale. The instability

355 of C-terminus is consistent with the previously reported result from generalized ensemble

356 simulations of the poly-Ala peptides (Young \& Brooks, 1996; Takano et al., 1999; Wu \& Wang,

357 2001). The mechanism of helix-coil transitions, shown here, might reflect the nature of the

358 peptide backbone, and provide insight into the helix-coil transitions for general cases of

359 polypeptides.

360

361 Acknowledgements

362 The supercomputer resources were provided by the HPCI System Research Projects (Project IDs:

$363 \mathrm{hp170020}$ and hp170025) and the National Institute of Genetics, Research Organization of

364 Information and Systems, Japan. We thank Tomoya Hirano for help with data analyses.

\section{REFERENCES}

Baldwin RL 1995. $\alpha$-Helix formation by peptides of defined sequence. Biophysical chemistry 55:127-135. DOI: 10.1016/0301-4622(94)00146-B.

369 Causgrove TP, Dyer RB 2006. Nonequilibrium protein folding dynamics: laser-induced $\mathrm{pH}$-jump

370 studies of the helix-coil transition. Chemical Physics 323:2-10. DOI:

$371 \quad$ 10.1016/j.chemphys.2005.08.032. 
372

373

374

375

376

377

378

379

380

381

382

383

384

385

386

387

388

389

390

391

392

393

394

395

396

397

398

399

400

401

402

403

404

405

406

407

408

409

410

411

412

413

414

415

416

417

Chen Y, Zhou Y, Ding J 2007. The helix-coil transition revisited. Proteins: Structure, Function, and Bioinformatics 69:58-68. DOI: 10.1002/prot.21492.

Clarke DT, Doig AJ, Stapley BJ, Jones GR 1999. The alpha-helix folds on the millisecond time scale. Proceedings of the National Academy of Sciences of the United States of America 96:7232-7237. DOI: 10.1073/pnas.96.13.7232.

Donten ML, Hamm P 2013. pH-jump induced $\alpha$-helix folding of poly-l-glutamic acid. Chemical Physics 422:124-130. DOI: 10.1016/j.chemphys.2012.11.023.

Finke JM, Jennings PA, Lee JC, Onuchic JN, Winkler JR 2007. Equilibrium unfolding of the poly(glutamic acid)20 helix. Biopolymers 86:193-211. DOI: 10.1002/bip.20719.

Gooding EA, Sharma S, Petty SA, Fouts EA, Palmer CJ, Nolan BE, Volk M 2013. pH-dependent helix folding dynamics of poly-glutamic acid. Chemical Physics 422:115-123. DOI: 10.1016/j.chemphys.2012.11.009.

Gómez-Sicilia À, Sikora M, Cieplak M, Carrión-Vázquez M 2015. An Exploration of the Universe of Polyglutamine Structures. PLoS computational biology 11:e1004541. DOI: 10.1371/journal.pcbi.1004541.

Greenfield NJ 2007. Using circular dichroism spectra to estimate protein secondary structure. Nature Protocols 1:2876-2890. DOI: 10.1038/nprot.2006.202.

Henriques J, Skepö M 2016. Molecular Dynamics Simulations of Intrinsically Disordered Proteins: On the Accuracy of the TIP4P-D Water Model and the Representativeness of Protein Disorder Models. Journal of Chemical Theory and Computation 12:3407-3415. DOI: $10.1021 /$ acs.jctc.6b00429.

Hornak V, Abel R, Okur A, Strockbine B, Roitberg A, Simmerling C 2006. Comparison of multiple Amber force fields and development of improved protein backbone parameters. Proteins: Structure, Function, and Bioinformatics 65:712-725. DOI: 10.1002/prot.21123.

Huang J, Rauscher S, Nawrocki G, Ran T, Feig M, de Groot BL, Grubmüller H, Mackerell AD 2016. CHARMM36m: an improved force field for folded and intrinsically disordered proteins. Nature Methods 14:71-73. DOI: 10.1038/nmeth.4067.

Inoue K, Baden N, Terazima M 2005. Diffusion Coefficient and the Secondary Structure of Poly-l-glutamic Acid in Aqueous Solution. The Journal of Physical Chemistry B 109:2262322628. DOI: $10.1021 /$ jp052897y.

Jorgensen WL, Chandrasekhar J, Madura JD, Impey RW, Klein ML 1983. Comparison of simple potential functions for simulating liquid water. The Journal of chemical physics 79:926-935. DOI: $10.1063 / 1.445869$.

Kabsch W, Sander C 1983. Dictionary of protein secondary structure: Pattern recognition of hydrogen-bonded and geometrical features. Biopolymers 22:2577-2637. DOI: 10.1002/bip.360221211.

Kasahara K, Sakuraba S, Fukuda I 2018. Enhanced Sampling of Molecular Dynamics Simulations of a Polyalanine Octapeptide: Effects of the Periodic Boundary Conditions on Peptide Conformation. The Journal of Physical Chemistry B 122:2495-2503. DOI: 10.1021/acs.jpcb.7b10830.

Kastenholz MA, Hünenberger PH 2004. Influence of Artificial Periodicity and Ionic Strength in Molecular Dynamics Simulations of Charged Biomolecules Employing Lattice-Sum Methods. The Journal of Physical Chemistry B 108:774-788. DOI: 10.1021/jp0350924.

Kelly SM, Jess TJ, Price NC 2005. How to study proteins by circular dichroism. Biochimica et Biophysica Acta (BBA) - Proteins and Proteomics 1751:119-139. DOI: 10.1016/j.bbapap.2005.06.005. 
418 Kimura T, Takahashi S, Akiyama S, Uzawa T, Ishimori K, Morishima I 2002. Direct

419

420

421

422

423

424

425

426

427

428

429

430

431

432

433

434

435

436

437

438

439

440

441

442

443

444

445

446

447

448

449

450

451

452

453

454

455

456

457

458

459

460

461

462

463

Observation of the Multistep Helix Formation of Poly- 1-glutamic Acids. Journal of the American Chemical Society 124:11596-11597. DOI: 10.1021/ja026639f.

Nakamura H, Wada A 1981. Dielectric studies of aqueous solutions of poly(L-glutamic acid). Biopolymers 20:2567-2582. DOI: 10.1002/bip.1981.360201207.

Neumaier S, Reiner A, Büttner M, Fierz B, Kiefhaber T 2013. Testing the diffusing boundary model for the helix-coil transition in peptides. Proceedings of the National Academy of Sciences 110:12905-12910. DOI: 10.1073/pnas.1303515110.

Pal L, Chakrabarti P, Basu G 2003. Sequence and Structure Patterns in Proteins from an Analysis of the Shortest Helices: Implications for Helix Nucleation. Journal of Molecular Biology 326:273-291. DOI: 10.1016/S0022-2836(02)01338-4.

Piana S, Donchev AG, Robustelli P, Shaw DE 2015. Water Dispersion Interactions Strongly Influence Simulated Structural Properties of Disordered Protein States. The Journal of Physical Chemistry B 119:5113-5123. DOI: 10.1021/jp508971m.

Piana S, Klepeis JL, Shaw DE 2014. Assessing the accuracy of physical models used in proteinfolding simulations: quantitative evidence from long molecular dynamics simulations. Current opinion in structural biology 24:98-105. DOI: 10.1016/j.sbi.2013.12.006.

Qin Z-J, Shimizu A, Li J, Ikeguchi M, Shinjo M, Kihara H 2014. $\alpha$-helix formation rate of oligopeptides at subzero temperatures. BIOPHYSICS 10:9-13. DOI: 10.2142/biophysics.10.9.

Reif MM, Kräutler V, Kastenholz MA, Daura X, Hünenberger PH 2009. Molecular Dynamics Simulations of a Reversibly Folding $\beta$-Heptapeptide in Methanol: Influence of the Treatment of Long-Range Electrostatic Interactions. The Journal of Physical Chemistry B 113:31123128. DOI: 10.1021/jp807421a.

Richardson JS 1981. The anatomy and taxonomy of protein structure. Advances in protein chemistry 34:167-339.

Ryckaert JP, Ciccotti G, Berendsen HJC 1977. Numerical integration of the cartesian equations of motion of a system with constraints: molecular dynamics of n-alkanes. Journal of Computational Physics 23:327-341. DOI: 10.1016/0021-9991(77)90098-5.

Sorin EJ, Pande VS 2005. Exploring the Helix-Coil Transition via All-Atom Equilibrium Ensemble Simulations. Biophysical Journal 88:2472-2493. DOI: 10.1529/biophysj.104.051938.

Spek EJ, Olson CA, Shi Z, Kallenbach NR 1999. Alanine Is an Intrinsic $\alpha$-Helix Stabilizing Amino Acid. Journal of the American Chemical Society 121:5571-5572. DOI: 10.1021/ja990056x.

Stanley CB, Strey HH 2008. Osmotically Induced Helix-Coil Transition in Poly(Glutamic Acid). Biophysical Journal 94:4427-4434. DOI: 10.1529/biophysj.107.122705.

Takano M, Yamato T, Higo J, Suyama A, Nagayama K 1999. Molecular Dynamics of a 15Residue Poly( 1-alanine) in Water: Helix Formation and Energetics. Journal of the American Chemical Society 121:605-612. DOI: 10.1021/ja982919c.

Takano Y, Kusaka A, Nakamura H 2016. Density functional study of molecular interactions in secondary structures of proteins. BIOPHYSICS 13:27-35. DOI: 10.2142/biophysico.13.0_27. Weber W, Hünenberger PH, McCammon JA 2000. Molecular Dynamics Simulations of a Polyalanine Octapeptide under Ewald Boundary Conditions: Influence of Artificial Periodicity on Peptide Conformation. The Journal of Physical Chemistry B 104:3668-3675. DOI: $10.1021 /$ jp9937757. 
464 Wu X, Wang S 2001. Helix Folding of an Alanine-Based Peptide in Explicit Water. The Journal of Physical Chemistry B 105:2227-2235. DOI: 10.1021/jp004048a.

466

467

468

469

470

471

472

473

Figure Legends

Young WS, Brooks CL 1996. A microscopic view of helix propagation: N and C-terminal helix growth in alanine helices. Journal of Molecular Biology 259:560-572. DOI:

474

Figure 1. The initial structures of MD simulations. (A) The structure built by a REMD simulation, termed Sim. (B) The structure based on the ideal $\alpha$-helix, termed Ide. (C) $\varphi-\psi$ angles 475 of 2nd-20th residues in Sim (triangles; the open triangles indicate the 2nd and 20th) and Ide 476 (circle; all residues have the same values).

477

Figure 2. The $3.0-\mu \mathrm{s}$ time courses of the secondary structure elements and examples of 478 snapshots for $\operatorname{Sim} 1, \operatorname{Sim} 2$, and $\operatorname{Sim} 3$ simulations. (A, B, and C) The time courses for $\operatorname{Sim} 1, \operatorname{Sim} 2$, and $\operatorname{Sim} 3$, respectively. The horizontal axis is the simulation time, and the vertical axis indicates the amino acid position in the peptide chain. Each block is filled by one of the eight types of colors regarding the secondary structure elements $H, G, I, E, B, T, S$, and $O$, are indicated as red, maroon, dark-red, gray, black, dark-cyan, cyan, and white, respectively. (D, E, F, G, and H) Snapshots at (D) $0.625 \mu \mathrm{s}$ in $\operatorname{Sim} 1$, (E) $0.804 \mu \mathrm{s}$ in $\operatorname{Sim} 1$, (F) $0.842 \mu \mathrm{s}$ in $\operatorname{Sim} 1$, (G) $0.199 \mu \mathrm{s}$ in $484 \operatorname{Sim} 2$, and (H) $1.000 \mu \mathrm{s}$ in $\operatorname{Sim} 2$.

Figure 3. The time course of helix content averaged over accumulated time duration of each 486 trajectory in $\operatorname{Sim} 1, \operatorname{Sim} 2$, and $\operatorname{Sim} 3$.

487 Figure 4. The 400-ns time courses of the secondary structure elements of Ide $1-20$ for the panels 488 (A)-(T), respectively. See the legend of Figures 2A, B, and C.

Figure 5. The 400-ns time courses of the secondary structure elements of $\operatorname{Sim} 1-8$ for the panels 
491 Figure 6. Residue-wise secondary structure content of $\alpha$-helix ( $H$; solid), $3_{10}$-helix ( $G$; dashed), 492 and turn conformations ( $T$; dotted). (A) The average over 20 Ide runs $\left(P^{I d e}(x ; i)\right)$. (B) The average 493 over the 400-ns trajectories of $8 \operatorname{Sim}$ runs $\left(P^{\operatorname{Sim}}(x ; i)\right)$. (C) The average over 3.0 - $\mu$ s trajectories of $494 \operatorname{Sim} 1, \operatorname{Sim} 2$, and $\operatorname{Sim} 3\left(P^{\operatorname{Sim} 1-3}(x ; i)\right)$.

495 Supplementary Figure S1. Time course of (A) end-to-end distance and (B) radius of gyration of 496 PGA in Sim1 (red), Sim2 (green), and Sim3 (blue) runs.

497 Supplementary Figure S2. Probabilities of transition between different secondary structures in 498 Ide runs. The horizontal axis indicates the position of each residue, $i$. Transitions from $\alpha$-helix $499(H), 3_{10}$-helix $(G)$, and turn $(T)$ are shown in solid, dashed, and dotted lines, respectively. 500 Destination states are indicated as red, maroon, dark-cyan, and green, for $H, G, T$, and structural 501 elements other than these, respectively. 


\section{Figure 1}

The initial structures of MD simulations.

(A) The structure built by a REMD simulation, termed Sim. (B) The structure based on the ideal $\alpha$-helix, termed Ide. (C) $\varphi$ - $\psi$ angles of 2 nd-20th residues in Sim (triangles; the open triangles indicate the 2 nd and 20th) and Ide (circle; all residues have the same values).

A

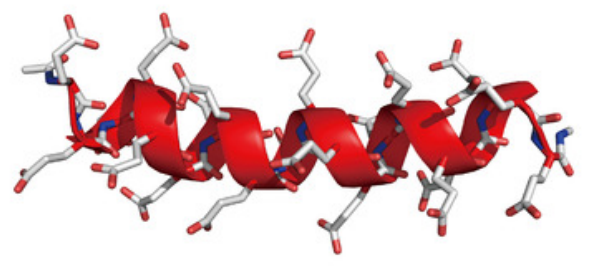

B

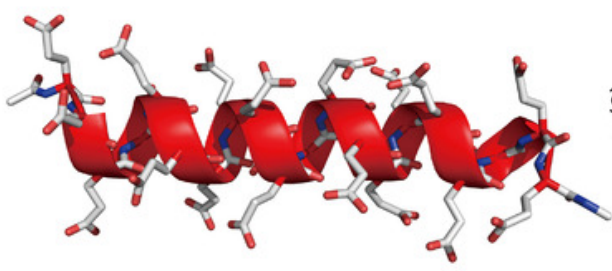

$c$
$\frac{7}{\ni}$

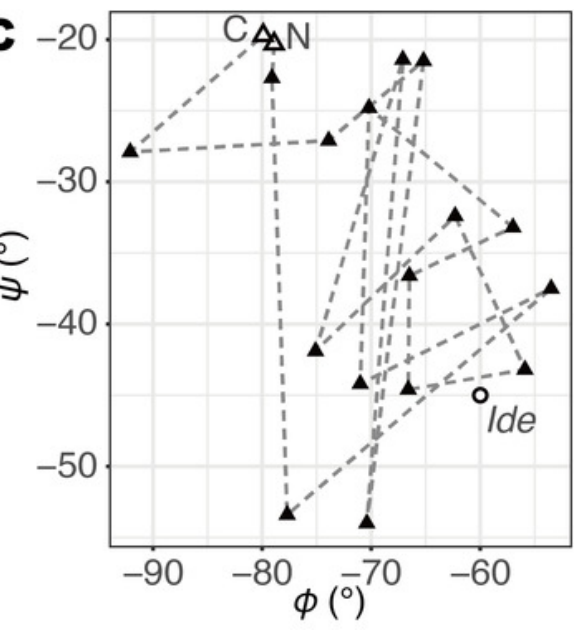




\section{Figure 2}

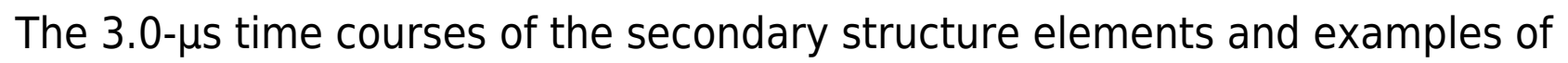
snapshots for $\operatorname{Sim} 1, \operatorname{Sim} 2$, and $\operatorname{Sim} 3$ simulations.

(A, B, and C) The time courses for $\operatorname{Sim} 1, \operatorname{Sim} 2$, and $\operatorname{Sim} 3$, respectively. The horizontal axis is the simulation time, and the vertical axis indicates the amino acid position in the peptide chain. Each block is filled by one of the eight types of colors regarding the secondary structure elements $H, G, I, E, B, T, S$, and $O$, are indicated as red, maroon, dark-red, gray, black, dark-cyan, cyan, and white, respectively. (D, E, F, G, and H) Snapshots at (D) $0.625 \mu \mathrm{s}$ in Sim1, (E) $0.804 \mu$ s in Sim1, (F) $0.842 \mu$ s in Sim1, (G) $0.199 \mu$ s in Sim2, and (H) $1.000 \mu s$ in $\operatorname{sim} 2$. 
A

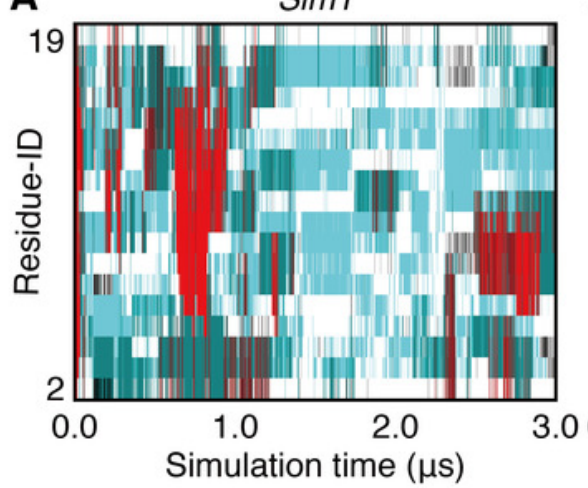

D

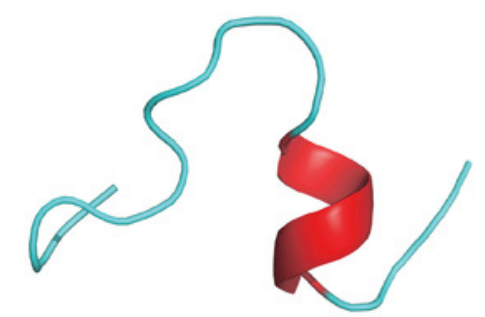

G

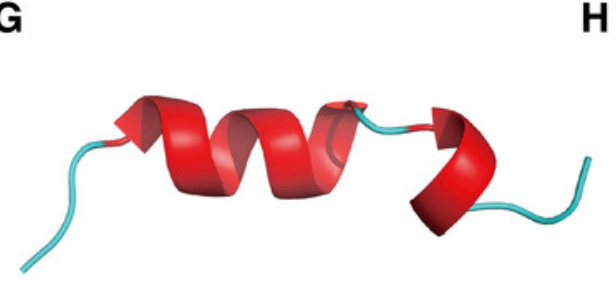

B

E

H
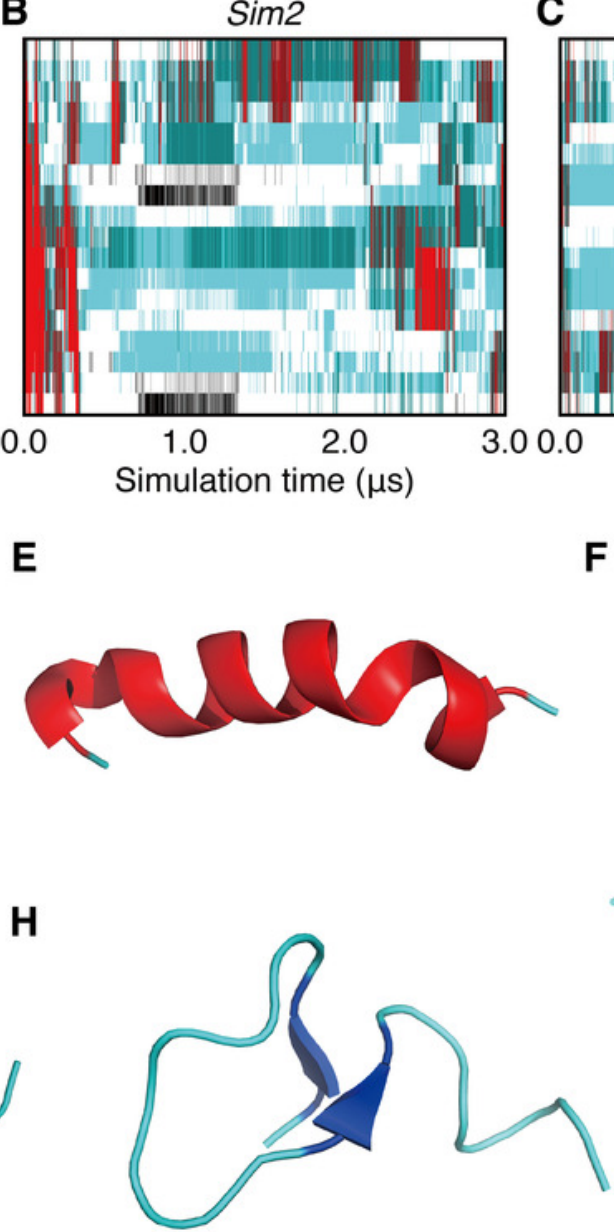

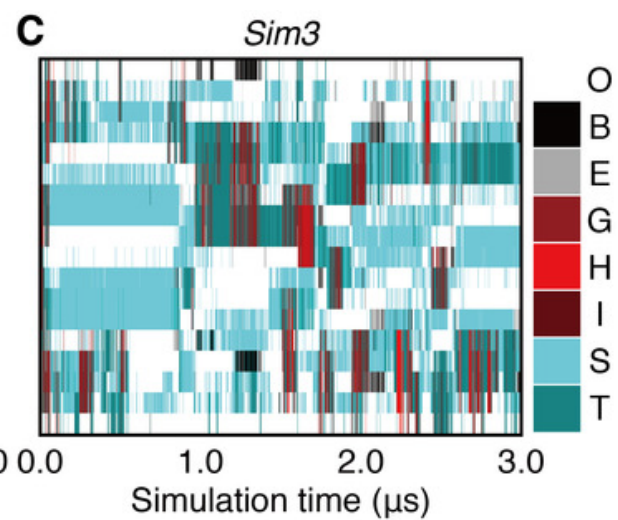

F

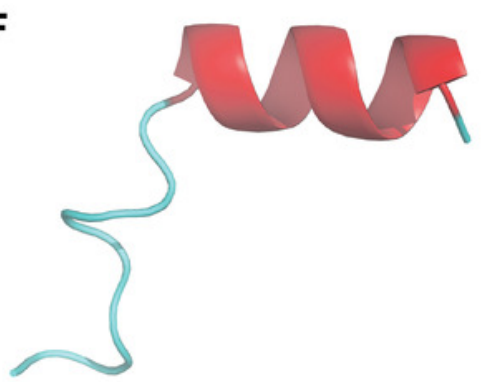


Figure 3

The time course of helix content averaged over accumulated time duration of each trajectory in Sim1, Sim2, and Sim3.

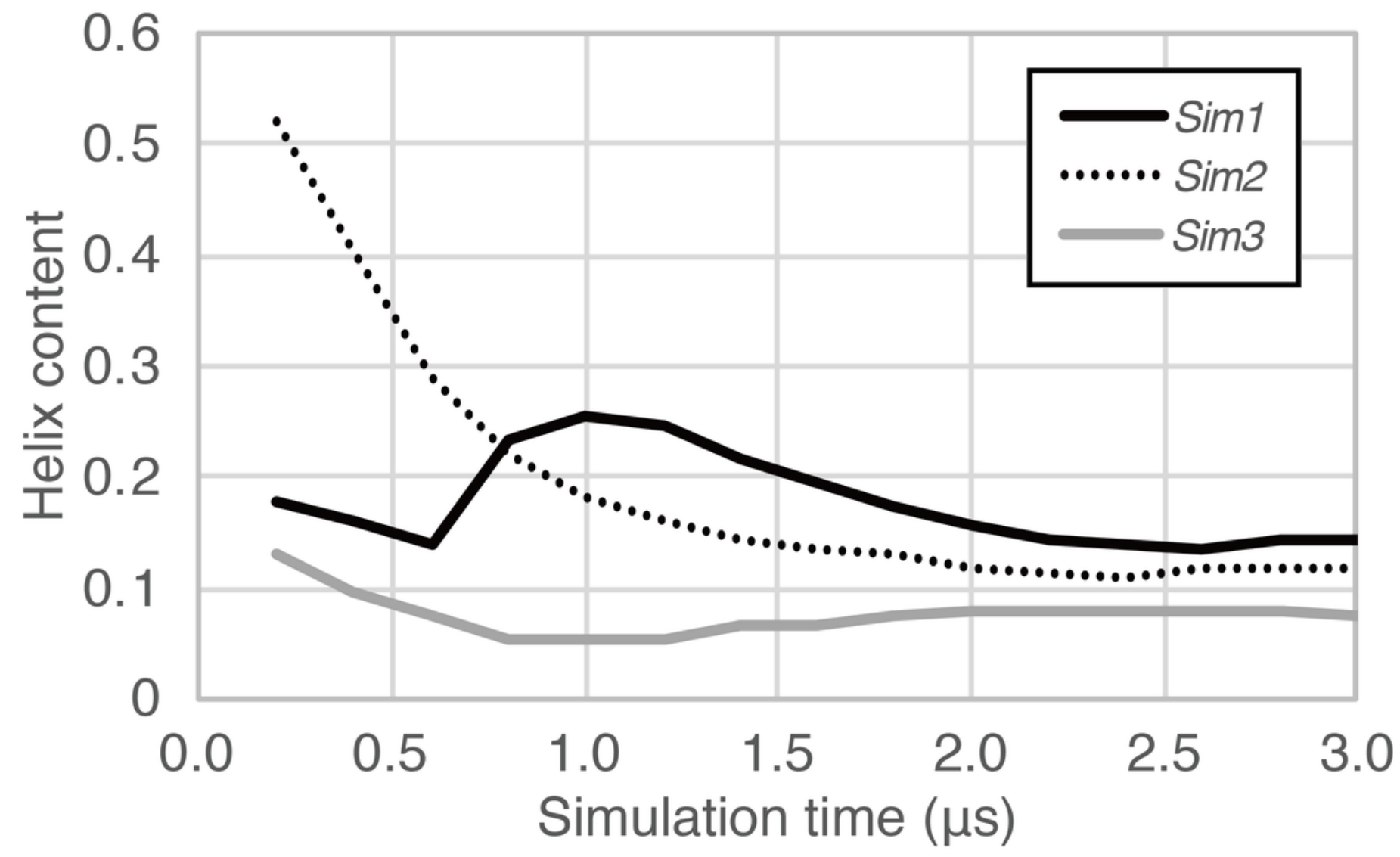




\section{Figure 4}

The 400-ns time courses of the secondary structure elements of Ide1-20 for the panels (A)-(T), respectively.

See the legend of Figures 2A, B, and C.

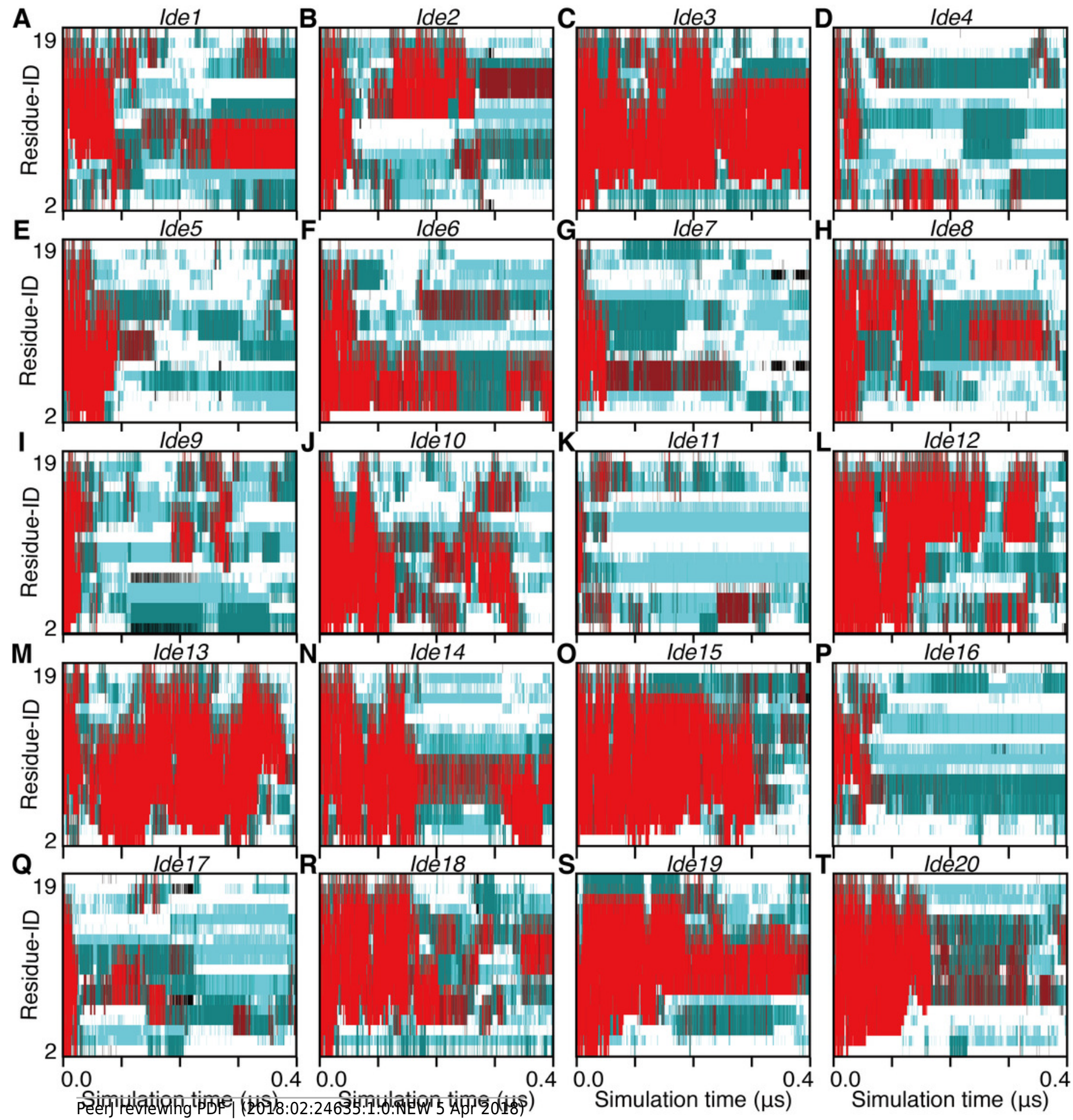


Figure 5

The 400-ns time courses of the secondary structure elements of Sim1-8 for the panels (A)-(H), respectively.

See the legend of Figures 2A, B, and C.
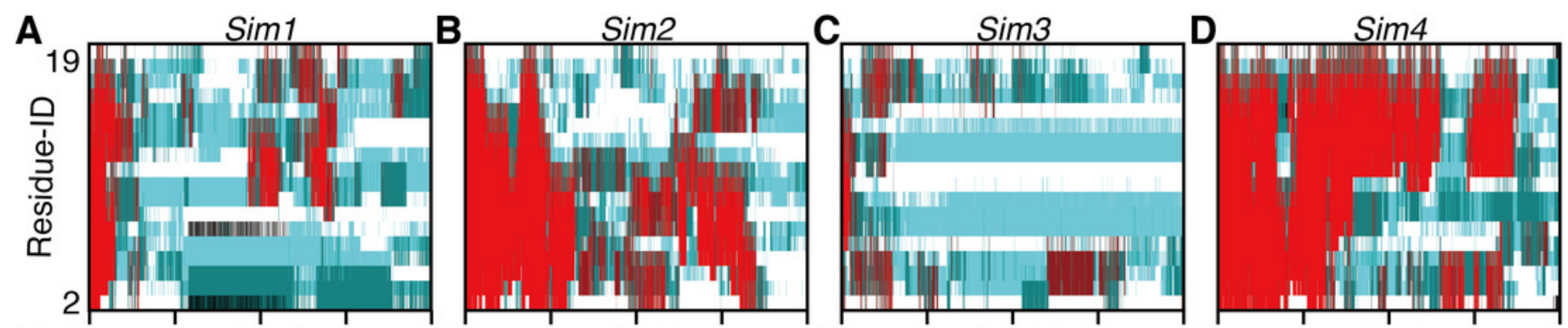

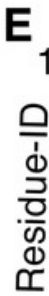
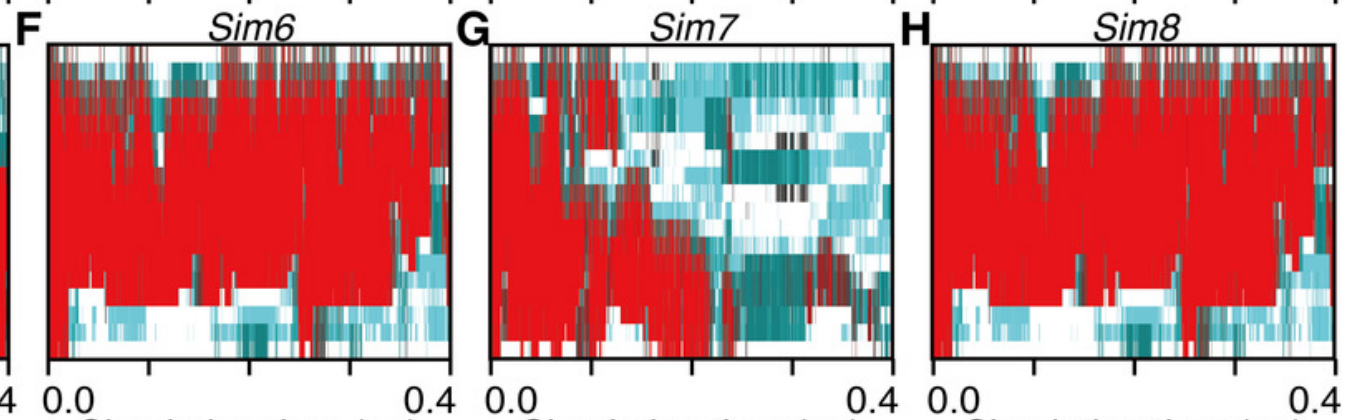

Simulation time $(\mu s)$

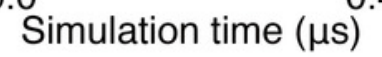

Simulation time $(\mu \mathrm{s})$

Simulation time $(\mu \mathrm{s})$ 
Figure 6

Residue-wise secondary structure content of $\alpha$-helix ( $H$; solid), $3_{10}$-helix ( $G$; dashed), and turn conformations ( $T$; dotted).

(A) The average over 20 Ide runs $\left(P^{\text {lde }}(x ; i)\right)$. (B) The average over the 400 -ns trajectories of 8

Sim runs $\left(P^{\operatorname{sim}}(x ; i)\right) .(C)$ The average over 3.0- $\mu$ s trajectories of Sim1, Sim2, and $\operatorname{Sim} 3$ $\left(P^{\operatorname{sim} 1-3}(x ; i)\right)$.
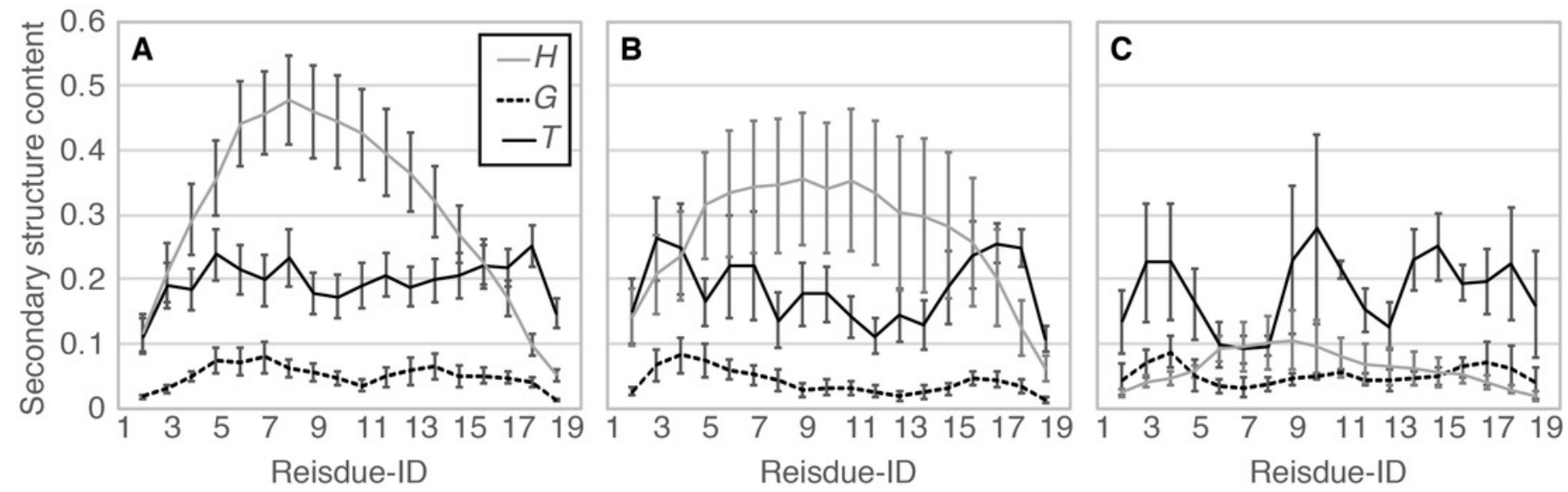
Table $\mathbf{1}$ (on next page)

Unfolding properties of each run. 
1 Table 1. Unfolding properties of each run.

\begin{tabular}{|c|c|c|c|}
\hline Run-ID & $t_{\mathrm{u}}$ & Unfolding order & $\mathrm{P}(\mathrm{H})+\mathrm{P}(\mathrm{G})$ \\
\hline Ide1 & 8.52 & $\mathrm{~N}, \mathrm{C}, \mathrm{M}$ & 0.34 \\
\hline Ide2 & 36.98 & $\mathrm{C}, \mathrm{N}, \mathrm{M}$ & 0.34 \\
\hline Ide3 & 88.26 & $\mathrm{C}, \mathrm{N}, \mathrm{M}$ & 0.64 \\
\hline Ide4 & 10.30 & $\mathrm{C}, \mathrm{M}, \mathrm{N}$ & 0.13 \\
\hline Ide 5 & 74.82 & $\mathrm{C}, \mathrm{M}, \mathrm{N}$ & 0.20 \\
\hline Ide6 & 47.62 & $\mathrm{C}, \mathrm{N}, \mathrm{M}$ & 0.32 \\
\hline Ide 7 & 18.10 & $\mathrm{C}, \mathrm{N}, \mathrm{M}$ & 0.15 \\
\hline Ide 8 & 40.42 & $\mathrm{C}, \mathrm{N}, \mathrm{M}$ & 0.30 \\
\hline Ide 9 & 23.88 & $\mathrm{C}, \mathrm{N}, \mathrm{M}$ & 0.14 \\
\hline Ide10 & 101.34 & $\mathrm{~N}, \mathrm{C}, \mathrm{M}$ & 0.47 \\
\hline Ide11 & 257.92 & $\mathrm{C}, \mathrm{N}, \mathrm{M}$ & 0.60 \\
\hline Ide12 & 16.32 & $\mathrm{C}, \mathrm{M}, \mathrm{N}$ & 0.30 \\
\hline Ide13 & 29.52 & $\mathrm{~N}, \mathrm{C}, \mathrm{M}$ & 0.62 \\
\hline Ide14 & 19.40 & $\mathrm{~N}, \mathrm{C}, \mathrm{M}$ & 0.45 \\
\hline Ide15 & 249.24 & $\mathrm{C}, \mathrm{N}, \mathrm{M}$ & 0.60 \\
\hline Ide16 & 13.02 & $\mathrm{~N}, \mathrm{C}, \mathrm{M}$ & 0.09 \\
\hline Ide 17 & 23.62 & $\mathrm{C}, \mathrm{N}, \mathrm{M}$ & 0.14 \\
\hline Ide 18 & 35.06 & $\mathrm{C}, \mathrm{N}, \mathrm{M}$ & 0.44 \\
\hline Ide19 & 192.74 & $\mathrm{~N}, \mathrm{C}, \mathrm{M}$ & 0.50 \\
\hline Ide20 & 165.86 & $\mathrm{~N}, \mathrm{C}, \mathrm{M}$ & 0.41 \\
\hline Sim1 & 31.06 & $\mathrm{C}, \mathrm{N}, \mathrm{M}$ & 0.15 \\
\hline $\operatorname{Sim} 2$ & 100.52 & $\mathrm{C}, \mathrm{N}, \mathrm{M}$ & 0.12 \\
\hline $\operatorname{Sim} 3$ & 7.38 & $\mathrm{C}, \mathrm{N}, \mathrm{M}$ & 0.08 \\
\hline $\operatorname{Sim} 4$ & 79.66 & $\mathrm{C}, \mathrm{M}, \mathrm{N}$ & 0.51 \\
\hline Sim5 & 24.38 & $\mathrm{C}, \mathrm{M}, \mathrm{N}$ & 0.22 \\
\hline Sim6 & 380.74 & $\mathrm{~N}, \mathrm{C}, \mathrm{M}$ & 0.65 \\
\hline $\operatorname{Sim} 7$ & 87.06 & $\mathrm{C}, \mathrm{N}, \mathrm{M}$ & 0.35 \\
\hline Sim8 & 23.22 & $\mathrm{C}, \mathrm{N}, \mathrm{M}$ & 0.08 \\
\hline
\end{tabular}

2 
Table 2 (on next page)

Probabilities of secondary structure transitions. 
1 Table 2. Probabilities of secondary structure transitions, $P^{I d e}(B, A ; i)$.

\begin{tabular}{ccccccccccccc}
\hline$i$ & \multicolumn{9}{c}{2} & \multicolumn{1}{c}{11} & \multicolumn{6}{c}{19} \\
\hline$x \backslash y$ & $H$ & $G$ & $T$ & $\overline{H G T}$ & $H$ & $G$ & $T$ & $\overline{H G T}$ & $H$ & $G$ & $T$ & $\overline{H G T}$ \\
\hline$H$ & 0.94 & 0.01 & 0.03 & 0.02 & 0.96 & 0.01 & 0.03 & 0.00 & 0.58 & 0.01 & 0.34 & 0.07 \\
$G$ & 0.08 & 0.60 & 0.22 & 0.10 & 0.15 & 0.55 & 0.28 & 0.02 & 0.04 & 0.50 & 0.32 & 0.14 \\
$T$ & 0.04 & 0.04 & 0.79 & 0.13 & 0.07 & 0.04 & 0.88 & 0.01 & 0.13 & 0.03 & 0.69 & 0.15 \\
$\overline{H G T}$ & 0.00 & 0.00 & 0.02 & 0.98 & 0.00 & 0.00 & 0.04 & 0.95 & 0.00 & 0.00 & 0.03 & 0.97 \\
\hline
\end{tabular}

2

3 


\section{Table 3(on next page)}

Probabilities of helix folding and unfolding in Ide runs.

${ }^{{ }_{1} 1}$ The $\mathrm{N}$-terminal region consisting of the 2 nd-7th residues. ${ }^{* 2}$ The middle region consisting of the 3 8th-13th residues. ${ }^{*}$ The C-terminal region consisting of the 14th-19th residues. 
1 Table 3. Probabilities of helix folding and unfolding in Ide runs.

\begin{tabular}{ccccc}
\hline & All & $\mathrm{N}^{* 1}$ & $\mathrm{M}^{* 2}$ & $\mathrm{C}^{* 3}$ \\
\hline$P^{\text {Ide }}(H, H H H)$ & 0.96 & 0.97 & 0.97 & 0.91 \\
$P^{\text {Ide }}(-, H H-)$ & 0.26 & 0.16 & 0.25 & 0.30 \\
$P^{\text {Ide }}(-,-H H)$ & 0.08 & 0.06 & 0.13 & 0.34 \\
$P^{\text {Ide }}(H, H--)$ & 0.23 & 0.24 & 0.22 & 0.24 \\
$P^{\text {Ide }}(H,--H)$ & 0.04 & 0.03 & 0.07 & 0.05 \\
$P^{\text {Ide }}(H, H-H)$ & 0.09 & 0.03 & 0.10 & 0.08 \\
$P^{\text {Ide }}(H,---)$ & 0.02 & 0.02 & 0.02 & 0.02
\end{tabular}

$2{ }^{* 1}$ The N-terminal region consisting of the 2 nd -7 th residues. ${ }^{* 2}$ The middle region consisting of the

3 8th-13th residues. ${ }^{* 3}$ The C-terminal region consisting of the 14th-19th residues.

4 